\title{
CONSTRUCTION OF ERROR CONTROL RUN LENGTH LIMITED CODES EXPLOITING SOME PARITY MATRIX PROPERTIES
}

\author{
Katarína Farkašová * - Peter Farkaš ${ }^{* *}$ - Martin Rakús ** \\ — Eugen Ružický ${ }^{*}$ — Adão Silva ${ }^{* * *}$ — Atílio Gameiro ${ }^{* * *}$
}

\begin{abstract}
Error control codes (ECC) as well as translation codes (TC) are used today in many different systems such as computer storages, communications systems and consumer electronic devices. ECC introduce redundancy into the encoded digital sequence in order to decrease the number of errors at output of its decoder [1]. TC introduce redundancy, in order to translate any digital sequence at the input of TC encoder to such output sequence, which fulfills constrains deduced from practical requirements. It is possible to construct codes, which have both of these properties, so called Transcontrol codes or their subclass error control run length limited (ECRLL) codes. In this manuscript a new approach to construction of EC-RLL codes is presented. The new construction is based on some parity check matrix properties of a linear binary block code from which the new EC-RLL code is obtained.
\end{abstract}

K e y w ords: error control codes (ECC), translation codes (TC), error control run length limited codes, modifier

\section{INTRODUCTION}

The motivation for the research of EC-RLL codes could be traced back to late eighties of 20-th century when more efficient line codes were needed for telecommunications systems. In [2] and [3] the authors proposed construction, which exploited disparity for selection of codewords of ECC in order to achieve DC free line codes. Deeper analysis and explanation of this technique and its connection with standard practice in line coding area (namely selecting codewords from more dictionaries using running disparity) was published in [4]. In [5] and [6] construction of EC-RLL trellis codes and general method for single error correcting EC-RLL codes were presented respectively. In [7] it was proposed to modify a linear error control block code into Transcontrol code without introduction of additional redundant symbols. Later in [8] further possibilities how to construct EC-RLL codes using modifiers based on properties of generator matrices of ECC were found. (In this publication also the name Transcontrol codes was used for the first time.) Later on thanks to this technique Convolutional codes [9], Turbocodes [10] and [11], Extended Hamming codes [12], extended Golay code [13] and Reed-Muller codes [14] with translation properties were found. Especially the industry producing storage systems motivated and still motivate the research of relatively complex EC-RLL codes based on turbo codes and LDPC codes. This research is documented by numerous publications, for example [15] - [19]. It is not possible to give detailed overview of all results in this area in this manuscript. The interested reader could get more information from [20] or IET/IEEE electronic database.

\section{NEW TRANSCONTROL CODES}

One known EC-RLL construction starts with selecting an error control code or family of error control codes with suitable generator matrices. Then modifiers are selected and applied in cascade with the original encoder and decoder, as illustrated in Fig. 1. The questions arises which error control codes have to be used for starting the construction and which modifiers are appropriate? The answer is contained in the following properties presented and proofed in [8]:

1. If the Hamming weight of all rows in generator matrix $\boldsymbol{G}_{k \times n}$ is even, then all codewords of the corresponding linear block code have even weight.

2. If all codewords of a linear block code have even length and also even weight, then the inversion of an odd number of symbols in all of them creates a new code in which no codeword has symbols which are all equal to zero or all equal to one.

The new approach is based on an idea how to rearrange columns of systematic generating matrix $\boldsymbol{G}_{k \times n}=$ $\left[\boldsymbol{I}_{k \times k} \boldsymbol{P}_{k \times(n-k)}\right]$, where $\boldsymbol{I}_{k \times k}$ is an identity matrix and $\boldsymbol{P}_{k \times(n-k)}$ is a parity matrix, in order to get a sub matrix, which has all rows with even Hamming weight and at the same time it will have an even number of columns.

\footnotetext{
* Institute of Applied Informatics, Faculty of Informatics, Pan-European University, Bratislava, Slovakia, ${ }^{* *}$ Institute of Telecommunications, Faculty of Electrical Engineering and Information Technology, Slovak University of Technology, Slovakia, p.farkas@ieee.org, *** Telecommunications Institute, University of Aveiro, Portugal
} 


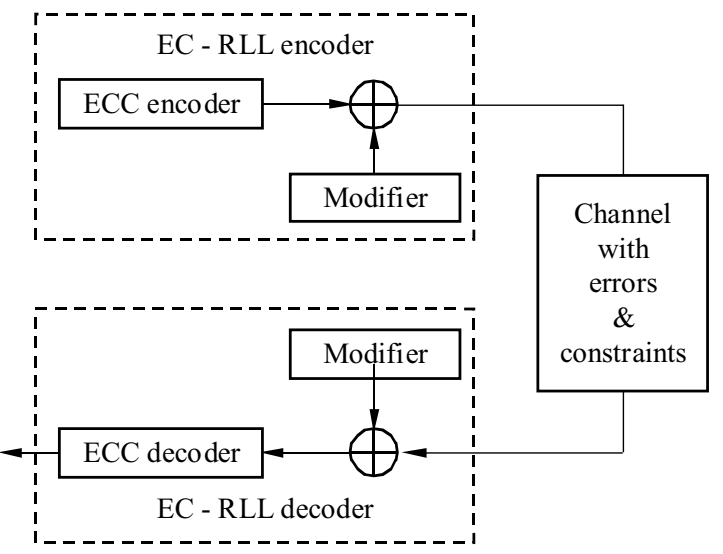

Fig. 1. 1 EC-RLL code obtained from an error control code using modifier (scrambler)

It has to be mentioned, that this rearrangement is applicable only to some systematic matrices of linear block codes. When this condition is satisfied the already known technique of modifier could be used to get an EC-RLL code as it is illustrated in Fig 1.

The rearrangement starts with the check whether the Hamming weight of rows of parity matrix $\boldsymbol{P}_{k \times(n-k)}$ is even or odd. Let denote the components from $G F(2)$ in matrix $\boldsymbol{P}_{k \times(n-k)}$ as follows

$$
\boldsymbol{P}_{k \times(n-k)}=\left[\begin{array}{cccc}
p_{1,1} & p_{1,2} & \cdots & p_{1,(n-k)} \\
p_{2,1} & p_{2,2} & \cdots & p_{2,(n-k)} \\
\vdots & \vdots & \cdots & \vdots \\
p_{k, 1} & p_{k, 2} & \cdots & p_{k,(n-k)}
\end{array}\right]
$$

The results of these calculations could be presented as elements of a column vector

$$
\begin{gathered}
\boldsymbol{\omega}^{\top}=\left(\omega_{1}, \quad \omega_{2}, \quad \cdots \quad \omega_{k}\right) \text { for } 1 \leq i \leq k \\
\omega_{i}=p_{i, 1}+p_{i, 2}+\cdots+p_{i,(n-k)}(\bmod 2) .
\end{gathered}
$$

After obtaining $\boldsymbol{\omega}^{\top}$, we can check this vector and decide how to perform the following step. There are several options:

(a) If $n$ is even and $\boldsymbol{\omega}^{\top}$ has all coordinates equal to 1 , than the original generating matrix $\boldsymbol{G}_{k \times n}$ fulfills the desired property (namely that all rows have even weight and is even). In such a case no rearrangements are needed and the modifier (denoted as $\mathbf{m}$ ) could be any vector of length $n$ and having any odd Hamming weight smaller than $n$.

(b) If $(n-k)$ is even and $\boldsymbol{\omega}^{\top}$ has all coordinates equal to 0 , than the original parity matrix $\boldsymbol{P}_{k \times(n-k)}$ fulfills the desired property (namely that all rows have even weight and $n$ is even). In such a case no rearrangements are needed and the modifier could be any vector with length and odd Hamming weight in $(n-k)$ coordinates corresponding to columns of $\boldsymbol{P}_{k \times(n-k)}$.

(c) If $(n-k)$ is odd and $\boldsymbol{\omega}^{\top}$ has odd Hamming weight than it is necessary to rearrange the columns of identity matrix in such a way that they will together with
$\boldsymbol{P}_{k \times(n-k)}$ form a sub-matrix $\boldsymbol{G}^{\prime}$. Sub-matrix $\boldsymbol{G}^{\prime}$ has all rows with even weight and number of columns (denoted as $\left.n^{\prime}\right)$ is defined as: $n^{\prime}=w\left(\boldsymbol{\omega}^{\top}\right)+(n-k)$. This rearrangement is quite simple, namely the col-umns from the identity sub-matrix with 1 in the same rows as are the nonzero coordinates of $\boldsymbol{\omega}$ can be placed immediately before or after or in between columns of $\boldsymbol{P}_{k \times(n-k)}$. We will denote this new matrix $\boldsymbol{M}$.

(d) If $(n-k)$ is even and $\boldsymbol{\omega}^{\top}$ has even Hamming weight than it is necessary to rearrange the columns of identity matrix in such a way that they will together with $\boldsymbol{P}_{k \times(n-k)}$ form a matrix $M$ with all rows with even weight and even length. This rearrangement is quite simple, namely the columns with 1 in the same rows as are the nonzero coordinates in $\boldsymbol{\omega}$ have to be placed immediately before or after or in between columns of $\boldsymbol{P}_{k \times(n-k)}$. We will for simplicity say into matrix $\boldsymbol{M}$.

(e) In case when $(n-k)$ is odd and $w\left(\boldsymbol{\omega}^{\top}\right)$ is even or $(n-k)$ is even and at the same time $w\left(\boldsymbol{\omega}^{\top}\right)$ is odd, than the construction based on rearrangement of columns of the identity matrix described in (a) to (d) will fail. However, it is still possible to try to get the desired properties of sub matrix $\boldsymbol{G}^{\prime}$. To do this it is necessary to find some columns of $\boldsymbol{P}_{k \times(n-k)}$, which could be moved outside from $\boldsymbol{G}^{\prime}$ in such a way that the remaining sub matrix of $\boldsymbol{P}_{k \times(n-k)}$ (denoted as $\left.\boldsymbol{P}^{\prime}\right)$ will together with its vector $w\left(\boldsymbol{\omega}^{\prime}\right)$ have such a properties that one of the cases (a) to (d) will hold.

(f) If the (e) cannot be finished successfully than it is still possible to try to find two or more sub matrices in $\boldsymbol{P}_{k \times(n-k)}$ which will fulfill the conditions (a) to (d) either separately or in groups. The search for this solution however will no more be as simple as in the cases (a) to (d) and it would be recommended to use computerized search.

(g) It is possible that any of the attempts following the approaches described in (a) to (f) will be successful. In such a case, it can be concluded that the original matrix $\boldsymbol{G}_{k \times n}$ is not suitable for the proposed construction and some other generating matrix $\boldsymbol{G}_{k \times n}$ has to be chosen for the next attempt. The new candidate matrix $\boldsymbol{G}_{k \times n}$ may have the same parameters $\left[n, k, d_{\min }\right]$, but generating a linear block code with different weight spectrum then had the original matrix $\boldsymbol{G}_{k \times n}$.

The RLL properties of the resulting code in case of success will guarantee that there will be not a longer run of identical symbols than [12].

\section{ONE EC-RLL CODE CONSTRUCTED USING THE NEW APPROACH}

We can chose a $[7,4,3]$ Hamming code, which has a $\boldsymbol{P}$ matrix defined by as

$$
\boldsymbol{P}=\left[\begin{array}{lll}
1 & 0 & 1 \\
1 & 1 & 1 \\
1 & 1 & 0 \\
0 & 1 & 1
\end{array}\right]
$$


and $\boldsymbol{\omega}^{\top}=(0100)$.

In other words, the parity check matrix $\boldsymbol{P}_{4 \times 3}$ has 3 columns (odd number) and $\boldsymbol{\omega}^{\top}$ has odd Hamming weight. This is the case (c) and it is necessary to rearrange the order of columns in the identity matrix. By closer observation, we can see that all rows of $\boldsymbol{P}$ have even Hamming except second row. Therefore it is necessary to change the order of columns in the identity matrix in such a way that the column vector $\mathbf{i}_{5}^{\top}=(0100)$ together with $\boldsymbol{P}_{4 \times 3}$ will form

$$
\boldsymbol{M}=\left[\begin{array}{llll}
0 & 1 & 0 & 1 \\
1 & 1 & 1 & 1 \\
0 & 1 & 1 & 0 \\
0 & 0 & 1 & 1
\end{array}\right]
$$

This matrix has the desired properties because it has even number of columns namely 4 and each row has even Hamming weight. The modifier could be for example the following vector $\mathbf{m}=(000000)$.

The resulting Transcontrol code will have code distance 3 , therefore it will be able to correct up to 1 error in a codeword and in any sequence of its codewords there will be no longer run of identical symbols than 12 .

\section{CONCLUDING REMARK}

In this manuscript a new approach for construction of EC-RLL codes from linear block codes was presented. The construction method was applied on one of the most popular ECC, namely binary [7, 4, 3] Hamming code as an example. From it a new EC-RLL code was obtained with no longer run of identical symbols than 12 .

\section{Acknowledgement}

This work was supported by, Slovak Research and Development Agency under contracts SK-AT-0020-12 and SK-PT-0014-12, by Scientific Grant Agency of Ministry of Education of Slovak Republic and Slovak Academy of Sciences under contract VEGA 1/0518/13, by EU RTD Framework Program under ICT COST Action IC 1104 and by Visegrad Fund and National Scientific Council of Taiwan under IVF - NSC, Taiwan Joint Research Projects Program no. 21280013 The Smoke in the Chimney - An Intelligent Sensor - based TeleCare Solution for Homes.

\section{REFERENCES}

[1] BLAHUT, R. E. : Principles and Practice of Information Theory, Addison Wesley, Reading/Mass, 1987.

[2] HERRO, M. A.-HU, L. : Error-Correcting Line Codes, in Proc. 23rd Ann. Allerton Conf. Communication, Control, and Computing, Monticello, IL, Oct 1985, pp. 450-451.
[3] DENG, R.-H.-HERRO, M. A.: DC-Free Coset Codes, Information Theory, IEEE Transactions on 34 No. 4 (July 1988), 786-792.

[4] POPPLEWELL, A.-O'REILLY, J.-J. "Spectral characteristics of a class of DC free error-correcting transmission codes," Electronics Letters, .

[5] YINYI LIN-WOLF, J. K. : Combined ECC/RLL Codes, Magnetics, IEEE Transactions on 24 No. 6 (Nov 1988), 2527-2529.

[6] LEE, P.-WOLF, J. K. : A General Error-Correcting Code Construction for Run-Length Limited Binary Channels, Information Theory, IEEE Transactions on 35 No. 6 (Nov 1989), 1330-1335.

[7] POPPLEWELL, A.-O'REILly, J.: Runlength Limited Binary Error Control Codes, Proc. IEE (1992), 349-355.

[8] FARKAS, P.-WEINRICHTER, H.: Transcontrol Codes with Run-Length Limitation, AEU Int. J. Electron. Commun. 50 (1996), 353-356.

[9] SECHNY, M.-FARKAS, P.: Some New Runlength-Limited Convolutional Codes, Communications, IEEE Transactions on 47 No. 7 (July 1999), 962-966.

10] FARKAS, P.-PUSCH, W.-TAFERNER, M.-WEINRICHTER, H. : Turbo-Codes with Length Constraints, AEÜ Internat. J. of Electronics and Communications 53 No. 3 (1999), 161-166.

[11] FARKAS, P.: Turbo-Codes with RLL Properties, In: IEE Colloquium Turbo Codes in Digital Broadcasting - Could it Double Capacity?, London, 22, Nov 1999, pp. 13/1-6.

12] CHOMIST, R.-FARKAS, P.: Some extended Hamming Transcontrol Codes, In: Radioelektronika 2005. 15-th International Czech-Slovak Scientific Conference, Brno, 3-4 May 2005, pp. $342-345$.

[13] CHOMIST, R.-FARKAS, P.: Extended Golay Code with Transcontrol Properties, In: 6th International Conference on Digital Signal Processing and Multimedia Communications. Proceedings of the DSP - MCOM 2005, Košice, Slovakia, 13-14 Sep 2005, pp. 102-105.

14] FARKAS, P.-CHOMIST, R.: Reed Muller-Codes with Run Length Properties, Mobile Future, 2004 and the Symposium on Trends in Communications. SympoTIC '04. Joint IST Workshop on, 24-26 Oct 2004, pp. 66-69.

[15] JUN LEE-JAEJIN LEE: Error Correcting RLL Codes USING High Rate RSC or Turbo Code, Electronics Letters 37 No. 17 (16 Aug 2001), 1074-1075.

[16] VASIC, B.-PEDAGANI, K.: Run-Length-Limited Low-Density Parity Check Codes based on Deliberate Error Insertion, Magnetics, IEEE Transactions on 40 No. 3 (May 2004), 1738-1743.

17] ZONGWANG LI-KUMAR, B. V. K. V.: Low-Density Parity-Check Codes with Run Length Limited (RLL) Constraints, Magnetics, IEEE Transactions on 42 No. 2 (Feb 2006), 344-349.

18] HONG-FU CHOU-YEONG-LUH UENG-MAO-CHAO LIN -FOSSORIER, M. P. C. : An RLL-Constrained LDPC Coded Recording System Using Deliberate Flipping and Flipped-Bit Detection, Communications, IEEE Transactions on 60 No. 12 (Dec 2012), 3587-3596.

[19] ARSLAN, S. S.-LEE, J.: Cycle Slip Detection and Correction through Classification of Run Length Limited Code Failures, Magnetics, IEEE Transactions on, accepted for publication, Apr 2013.

[20] IMMINK, K. A. S.: Codes for Mass Data Storage Systems, Shannon Foundation Publishers, Eindhoven, The Netherlands, 2004.

Received 1 December 2014 